\title{
Object Modeling using a ToF Camera under an Uncertainty Reduction Approach
}

\author{
Sergi Foix, Guillem Alenyà, Juan Andrade-Cetto and Carme Torras
}

\begin{abstract}
Time-of-Flight (ToF) cameras deliver 3D images at 25 fps, offering great potential for developing fast object modeling algorithms. Surprisingly, this potential has not been extensively exploited up to now. A reason for this is that, since the acquired depth images are noisy, most of the available registration algorithms are hardly applicable. A further difficulty is that the transformations between views are in general not accurately known, a circumstance that multi-view object modeling algorithms do not handle properly under noisy conditions. In this work, we take into account both uncertainty sources (in images and camera poses) to generate spatially consistent 3D object models fusing multiple views with a probabilistic approach. We propose a method to compute the covariance of the registration process, and apply an iterative state estimation method to build object models under noisy conditions.
\end{abstract}

\section{INTRODUCTION}

The interest for Time-of-Flight (ToF) cameras is rapidly growing thanks to the latest improvements in technology, resulting in contributions to diverse fields such as robot navigation, obstacle avoidance, human-machine interfaces, and particularly also in object modeling. A competing sensing technology for object modeling is lidar scanning, due to its precision, but which requires mobile parts to aggregate linear readings into full $3 \mathrm{D}$ scans with the subsequent detriment in frame rate. Stereo vision systems have also been used for object modeling, but require objects to be textured. On the contrary, ToF cameras offer registered depth-intensity images at high frame rate. Additionally, they offer other technical advantages such as robustness to illumination changes, low power consumption and low weight. However, the resolution of ToF camera images is typically low (i.e., $160 \times 120$ ), and depth values are much noisier compared to those of a laser scanner.

Object modeling has particular characteristics that require special attention when compared to other applications, e.g. scene modeling. First, the distance from the camera to the object is relatively small, so saturation in the center of the depth image and distortion are expected to appear. In addition, only part of the image corresponds to the target object and segmentation algorithms are required to perform background/foreground identification.

This work has been partially supported by the Spanish Ministry of Science and Innovation under project DPI2008-06022, the MIPRCV Consolider Ingenio 2010 project, and the EU PACO PLUS project FP6-2004-IST-427657. S. Foix and G. Alenyà are supported by $\mathrm{PhD}$ and postdoctoral fellowships, respectively, from CSIC's JAE program.

The authors are with the Institut de Robòtica i Informàtica Industrial, CSIC-UPC, Llorens i Artigas 4-6, 08028 Barcelona, Spain (e-mails: sfoix, galenya, cetto, torras@iri.upc.edu).

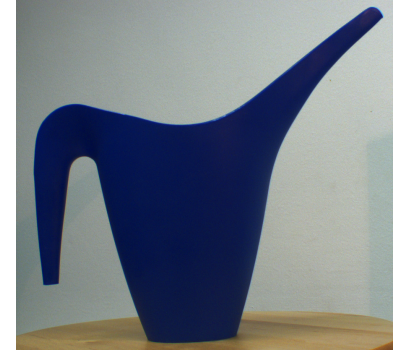

(a) Image of a watering can.

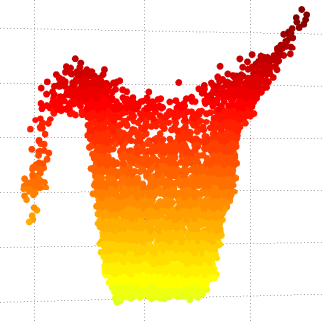

(b) 3D point cloud model
Fig. 1: A target object and its 3D point cloud model built using a Swissranger ToF camera with the proposed uncertainty-reduction approach. The 3D model maintains the object's surface topology, although sensor level noise in the individual points is still present.

In this paper we propose a method to compute the covariance of the registration process, and apply an iterative view aggregation method to build object models under noisy conditions (see Fig. 1). In our approach, views are taken along an approximately circular path around the object, and loop closure conditions are enforced at the end of the trajectory, significantly reducing the uncertainty in estimation.

Object modeling using ToF cameras has been addressed in comparison with stereo rig systems, with special emphasis on planar untextured surfaces [1], difficult background segmentation [2], geometric modeling and patchlet identification and pose estimation [3]. In fact, $\mathrm{ToF}$ and stereo systems naturally complement one another, and fusion algorithms have been proposed for the joint calibration of ToF and color cameras to obtain dense colored maps [4], [5], and object identification [6]. Another method to compute object models from ToF range data is based in coarse registration of point clouds with noise reduction and elimination of outliers by treating point sets as interacting masses connected via elastic forces [7].

We are interested in producing object models for humanoid robot object recognition and manipulation. Thus, models are required to preserve their surface topology. Moreover, it is desirable that models are acquired in the shortest time possible to permit real-time robot interaction with unknown objects. Less important for us is the precision in modeling the surface patches, as several surface smoothing algorithms are available that allow to extract geometric primitives useful for object grasping and manipulation once the model is built [8].

In the context of robotic manipulation, single ToF camera 
images have been used in the past to evaluate grasping properties such as force closure or obstacle avoidance [9]. In this work, we are interested in building a more complete reconstruction of objects, in terms of the surfaces bounding it, by combining multiple 3D depth images. The setup includes a ToF camera mounted on the end-effector of a robotic arm performing a circular trajectory around the object to acquire equally spaced images. These images could be combined via precise camera calibration w.r.t. the end effector and proper inverse kinematics of the manipulator, or alternatively, with a point cloud registration algorithm; usually a variant of the Iterative Closest Point (ICP) algorithm. Note that point cloud registration is more critical in object modeling than in scene modeling given the high signal-to-noise ratios of ToF depth information at relatively short distances. Moreover, for precisely calibrated robot-camera systems, the kinematics of humanoid robot arms is usually not very precise, and point cloud registration is still needed. Lastly, the successive registration of consecutive views accumulates drift error along a sequence. A common consequence is, for example, to compute the model of a circular object as a spiral. Thus, proper techniques for range data registration along multiple views need to be used.

Data fusion for scene or model augmentation has been typically addressed by error minimization methods such as bundle adjustment [10] or structure from motion [11]. These approaches are often not suitable for real time computation given their iterative nature. Recursive state estimation (e.g., SLAM) is a more suitable choice. The classical EKF-based approach to SLAM for feature-based scene augmentation is also not viable for real time modeling since it requires the computation of fully correlated covariances at each step [12]. In this work we propose to use a view-based informationform SLAM method that a) does not maintain a large number of feature estimates, but only a reduced number of pose estimates, and b) is efficiently computed in information form, exploiting the sparsity of such filtering representation [13]. We take advantage of the fact that the first and last images in a circular sequence around an object overlap. This allows us to impose a loop-closure constraint.

The rest of the article is structured as follows. In Section II the characteristics of ToF cameras are briefly discussed. Next, our modeling algorithm is presented in Section III, including the computation of the object pose resulting from the registration of point clouds and the estimation of the covariance of this pose (Sec. III-A) and the use of this covariance in model construction (Sec. III-B). Some experiments are presented in Section IV to evaluate the proposed covariance propagation algorithm, as well as the modeling algorithm. Finally, Section V is devoted to conclusions and prospects for future work.

\section{TOF CAMERAS}

ToF cameras emit modulated light, and depth computation is based on timed measures of the reflected light signal [14]. The modulation frequency of the emitted light determines the ambiguity-free range of the sensor, usually between 5 and 7

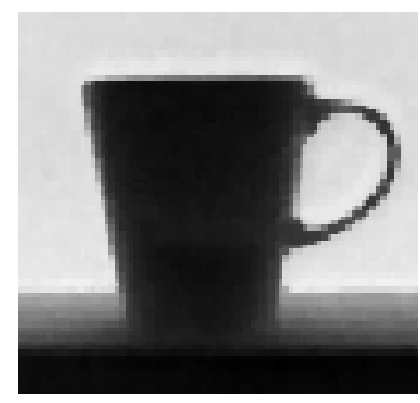

(a) 2D range image

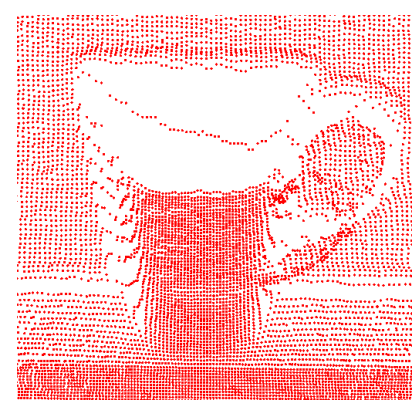

(b) Rotated 3D point cloud
Fig. 2: Typical output of a ToF camera. False depth readings appear at the edges between foreground and background due to the integration of the reflected light of both surfaces in the corresponding pixels.

meters. In comparison with other technologies also used to obtain scene depth, ToF cameras provide some interesting features:

1) registered dense depth and intensity images,

2) complete image acquisition at high frame rate,

3) small, low weight and compact design,

4) no mobile parts needed,

5) auto-illumination,

6) low power consumption.

Their main limitations are low resolution $(176 \times 144$ pixels for a SR3K camera) and noisy depth measurements, which are affected by both systematic and non-systematic errors. In general, systematic errors are dealt with calibration, whereas the effects of non-systematic errors is reduced with filtering. ToF camera calibration is still an active research field where some work has been done to completely understand the sources of errors and how to compensate them.

Systematic errors can be grouped into two categories. On the one hand, a correction for the depth value should be performed depending on the position of the pixel in the image matrix. This is generally calibrated using a known object and computing a suitable correction matrix, called sometimes fixed pattern noise matrix. On the other hand, a bias is introduced for each pixel that depends only on the measured depth. This bias is sinusoidal and it must be calibrated by taking measures of a known object at different known depths. B-splines are used to model this error in the general case, but if the range of depths is known to be restricted, a polynomial can be used instead.

One of the parameters that can be tuned in a ToF camera is the integration time, which has direct relation to the range of depths that the camera senses better. The calibration procedures mentioned above should be repeated for each different integration time used. Temperature can also affect the obtained depth. The most common strategy is to wait until the camera has reached a stable temperature value, and limit its use to a suitable operation range.

Non-systematic errors appear mainly due to problems in the reflection of light at concavities, edges (see Fig. 2), and in non-uniformly illuminated scenes. Furthermore, like 
for traditional cameras and depending on scene conditions, motion blur may appear if the camera acquires images during motion.

Our experiments are carried out using a fixed integration time and a low reflective object in order to maintain constant the systematic integration time-related error and to minimize unfavorable non-systematic specularities. Any integration time can be chosen as long as it does not distort the geometry of the scene. No other calibration approach has been used besides the mentioned parametrization.

\section{OBJECt MODELING FROM THE Accumulation of Point Clouds}

Each iteration of the proposed method comprises two steps. First, consecutive point clouds are registered using the ICP algorithm. The result includes estimates of the relative change in sensor pose between the two point clouds. To compute the covariance of the relative pose change, the sensor covariance is linearly propagated through the ICP minimization. The second step uses these first and second order camera pose change estimates to smooth the sensor motion sequence using a view-based SLAM method. The revised motion sequence is used to synthesize the final object model from the original views. The method is detailed in algorithmic form in Alg. 1, and each one of these steps is explained in more detail in the subsequent sections.

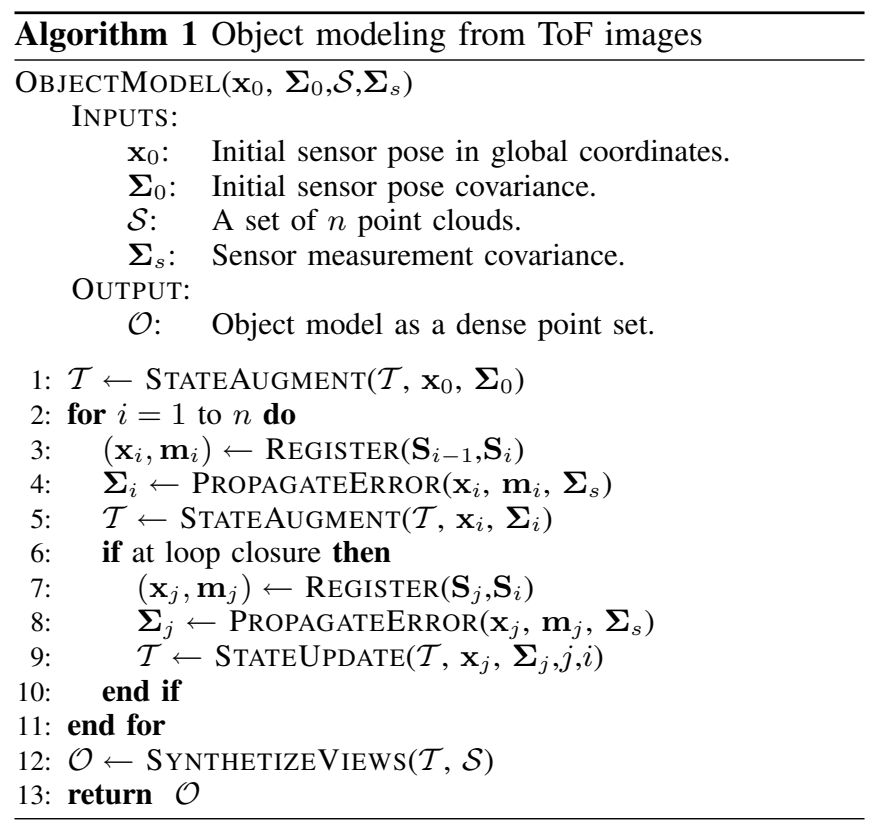

$\mathcal{T}$ represents the smoothed sensor pose history; $\mathbf{m}_{i}$ indicates the point correspondences between consecutive point clouds $i-1$ and $i ; \mathbf{m}_{j}$ indicates the point correspondences between loop-closing point clouds $j$ and $i ; \boldsymbol{\Sigma}_{i}$ is used to indicate the covariance of the relative pose change between consecutive point clouds $i-1$ and $i$; and $\boldsymbol{\Sigma}_{j}$ is used to indicate the covariance of the relative pose change between loop-closing point clouds $j$ and $i$.

\section{A. ICP Error Propagation}

The point cloud registration method used in this paper is based on the well-known ICP algorithm [15], [16], [17], and its variants [18], [19]. The probabilistic data fusion mechanism used in this work requires first order approximations of error propagation. That is, covariance estimates of sensor uncertainty must be propagated through the ICP cost function to compute relative pose covariance estimates between the two generative viewpoints.

The decision of using one cost function or another plays an important role during error propagation, since its derivatives need to be computed. In its simplest form, given a set of matching points from two consecutive point clouds $\mathbf{m}_{i}=$ $(a, b)$, the ICP cost function takes the form

$$
\varepsilon\left(\mathbf{m}_{i}, \mathbf{x}_{i}\right)=\sum\left\|\mathbf{x}_{i}(b)-a\right\|^{2} .
$$

An accurate covariance approximation can be computed using a Monte Carlo simulation, but this is a time-consuming solution and, since speed of execution is really a needed characteristic, finding a closed-form solution is desirable.

Given that the ICP algorithm is basically a cost function minimization procedure, an implicit function between input (point clouds) and the output (the pose) is defined by the minimization process [20]. Albeit the implicit function can not be explicitly known, its Jacobian matrix can be computed. Consequently, the estimated covariance matrix can be computed using the usual first-order approximation of an explicit function

$$
\boldsymbol{\Sigma}_{i}=\nabla \mathbf{f} \boldsymbol{\Sigma}_{s} \nabla \mathbf{f}^{\mathrm{T}}
$$

where $\nabla \mathbf{f}$ is the explicit function's Jacobian matrix, $\boldsymbol{\Sigma}_{s}$ the sensor covariance matrix and $\boldsymbol{\Sigma}_{i}$ the computed relative pose covariance matrix.

The Jacobian matrix of the ICP implicit function can be computed by means of the implicit function theorem. In our case, for an unconstrained minimization problem, the Jacobian matrix becomes

$$
\nabla \mathbf{f}=\left(\frac{\partial^{2} \varepsilon}{\partial \mathbf{x}_{i}^{2}}\right)^{-1} \frac{\partial^{2} \varepsilon}{\partial \mathbf{m}_{i} \partial \mathbf{x}_{i}}
$$

Since our approach uses the point to point Euclidean distance error as a cost function in the registration process, the application of the implicit function theorem is straight forward. It is important to notice however that this type of approximation propagates the error from sensor measurements to the sensor's relative pose. Therefore, the parametrization of the cost function will have to include the real sensor measurements as its only input variables. For instance, if a point-to-plane ICP algorithm is used, its point-to-plane function will have to be accommodated into the implicit function and derived consequently. It is not correct to precompute the virtual point of the plane correspondence and then apply a point-to-point cost function.

To evaluate the quality of our closed form covariance approximation, a Monte Carlo simulation was realized and the results were compared. Fig. 3 shows the results from 
the Monte Carlo simulation and the different closed-form approaches tried in Section IV-A.

\section{B. Closing the Loop}

Feature-based SLAM approaches are not adequate for fast object modeling due to the huge amount of data provided by the ToF camera, and the need to select a sparse feature representation. In our application, a view-based SLAM approach is used instead that optimizes only the relative sensor poses between views and their covariances. From the estimated history of sensor poses an object model is then synthesized using all sensor data. The final result is a finely registered dense point cloud which can be computed on-line.

The view-based SLAM technique used in our experiments is based on a delayed-state information-form algorithm [21], [13]. This algorithm, in contrast to feature-based SLAM approaches, has the advantage that the ensuing information matrix is naturally sparse and does not need extra sparsification steps that induce estimation errors.

The sensor pose (the $i$-th component of the state vector $\mathbf{x}$ ) contains the position of the sensor and its orientation in Euler angles $\mathbf{x}_{i}=\left[x_{k}, y_{k}, z_{k}, \phi_{k}, \theta_{k}, \psi_{k}\right]^{\mathrm{T}}$. The noise-free motion model is defined using the compounding operation [22], and defines the state transition model, relating state components $\mathbf{x}_{i-1}$ and $\mathbf{x}_{i}$,

$$
\begin{aligned}
\mathbf{x}_{i} & =f\left(\mathbf{x}_{i-1}, \mathbf{u}_{i}\right) \\
& =\mathbf{x}_{i-1} \oplus \mathbf{u}_{i},
\end{aligned}
$$

and $\mathbf{u}_{i}$ is the relative motion between consecutive poses as computed with the ICP algorithm.

We resort to the canonical parametrization of Gaussian distributions,

$$
\begin{gathered}
p(\mathbf{x})=\mathcal{N}(\mathbf{x} ; \boldsymbol{\mu}, \mathbf{\Sigma})=\mathcal{N}^{-1}(\mathbf{x} ; \boldsymbol{\eta}, \boldsymbol{\Lambda}), \\
\boldsymbol{\Lambda}=\mathbf{\Sigma}^{-1}, \text { and } \boldsymbol{\eta}=\mathbf{\Sigma}^{-1} \boldsymbol{\mu},
\end{gathered}
$$

where $\boldsymbol{\mu}$ is the mean state vector and $\boldsymbol{\Sigma}$ its covariance matrix, and $\boldsymbol{\Lambda}$ and $\boldsymbol{\eta}$ are the information matrix and information vector, respectively.

During state augmentation (line 5 in the Algorithm), the parameters $\boldsymbol{\eta}$ and $\boldsymbol{\Lambda}$ are computed with

$$
\boldsymbol{\eta}_{i-1, i}=\overline{\boldsymbol{\eta}}_{i-1, i}+\mathbf{F}_{\mathrm{aug}}^{\mathrm{T}} \boldsymbol{\Sigma}_{u}^{-1}\left(f\left(\boldsymbol{\mu}_{i-1}, \mathbf{u}_{i}\right)-\mathbf{F} \boldsymbol{\mu}_{i-1}\right)
$$

and

$$
\Lambda_{i-1: i, i-1: i}=\bar{\Lambda}_{i-1: i, i-1: i}+\mathbf{F}_{\text {aug }}^{\mathrm{T}} \boldsymbol{\Sigma}_{u}^{-1} \mathbf{F}_{\text {aug }},
$$

in which $\mathbf{F}_{\text {aug }}=\left[\begin{array}{ll}-\mathbf{F} & \mathbf{I}\end{array}\right], \mathbf{F}$ is the Jacobian matrix of the composition (Eq. 4), and $\overline{\boldsymbol{\eta}}_{i-1: i}$ and $\overline{\boldsymbol{\Lambda}}_{i-1: i, i-1: i}$ represent the posterior information vector and information matrix for poses $i-1$ and $i$, with zero entries for time $i$, indicating infinite uncertainty for that robot pose. The shared information between the new pose $\mathbf{x}_{i}$ and the rest of the robot trajectory $\mathbf{x}_{0: i-2}$ is always zero when we have not closed any loop. The result is a naturally sparse information matrix with a tridiagonal block structure.
Loop closures are also modeled using compounding operations,

$$
\begin{aligned}
\mathbf{z} & =h\left(\mathbf{x}_{j}, \mathbf{x}_{i}\right) \\
& =\ominus \mathbf{x}_{i} \oplus \mathbf{x}_{j},
\end{aligned}
$$

and the state update (line 9 in the Algorithm) is computed in information form with

$$
\begin{gathered}
\boldsymbol{\eta}_{j, i}=\overline{\boldsymbol{\eta}}_{j, i}+\mathbf{H}^{\mathrm{T}} \boldsymbol{\Sigma}_{z}^{-1}\left(\mathbf{z}-h\left(\boldsymbol{\mu}_{j}, \overline{\boldsymbol{\mu}}_{i}\right)+\mathbf{H} \overline{\boldsymbol{\mu}}_{j, i}\right) \\
\boldsymbol{\Lambda}_{j: i, j: i}=\overline{\boldsymbol{\Lambda}}_{j: i, j: i}+\mathbf{H}^{\mathrm{T}} \boldsymbol{\Sigma}_{z}^{-1} \mathbf{H},
\end{gathered}
$$

where $\mathbf{z}$ is the ICP's computed relative pose measurements between the current pose $\mathbf{x}_{i}$ and any other pose $\mathbf{x}_{j}$.

In the same way as with the prediction step, given the twoblock size of the measurement Jacobian matrix $\mathbf{H}$ (the partial derivative of Eq. 9), only the four blocks relating poses $j$ and $i$ in the information matrix will be updated.

Motivated by [23], we employ a QR factorization of the information matrix to solve $\boldsymbol{\Lambda} \boldsymbol{\mu}=\boldsymbol{\eta}$ and $\boldsymbol{\Lambda} \boldsymbol{\Sigma}=\mathbf{I}$, for $\boldsymbol{\mu}$ and $\boldsymbol{\Sigma}$.

In order to reduce the fill-in in the right triangular matrix from the QR factorization, we first reorder the information matrix using the column approximate minimum degree (COLAMD) ordering [24], then we apply QR factorization to the reordered information matrix and solve for each state variable via back substitution. State recovery takes nearly linear time, compatible with other state of the art SLAM approaches [25], [13].

\section{EXPERIMENTS}

In order to evaluate the proposed covariance propagation method a comparison with two other approaches to covariance estimation is presented: a) a Monte Carlo simulation, and b) the naive technique of aggregating matching distance errors between the point clouds. Furthermore, a modeling experiment using real data is also presented, showing the advantages of the proposed method with respect to the use of aggregated ICP in scenarios where both camera motion estimation and depth measurements are noisy.

\section{A. Monte Carlo Simulation}

To synthetically simulate the form of the relative pose covariance computed from ICP registration, a uniformly distributed point cloud is first generated with 105 points separated about $30 \mathrm{~mm}$ from each other along the three axes. A thousand secondary matching point clouds are generated from the same set of points, by applying a known rigid body transformation, and adding zero mean white noise with $5 \mathrm{~mm}$ standard deviation to each resulting data point, simulating sensor induced depth range measurement error. To avoid errors induced by unreliable nearest neighbor computation, point correspondences are given. On a side note, finding a good set of matching points is a critical part of the ICP algorithm. That is the reason why two filters have been implemented for the case of real data in order to increase its robustness. The first one is an outlier filter, guaranteeing good 

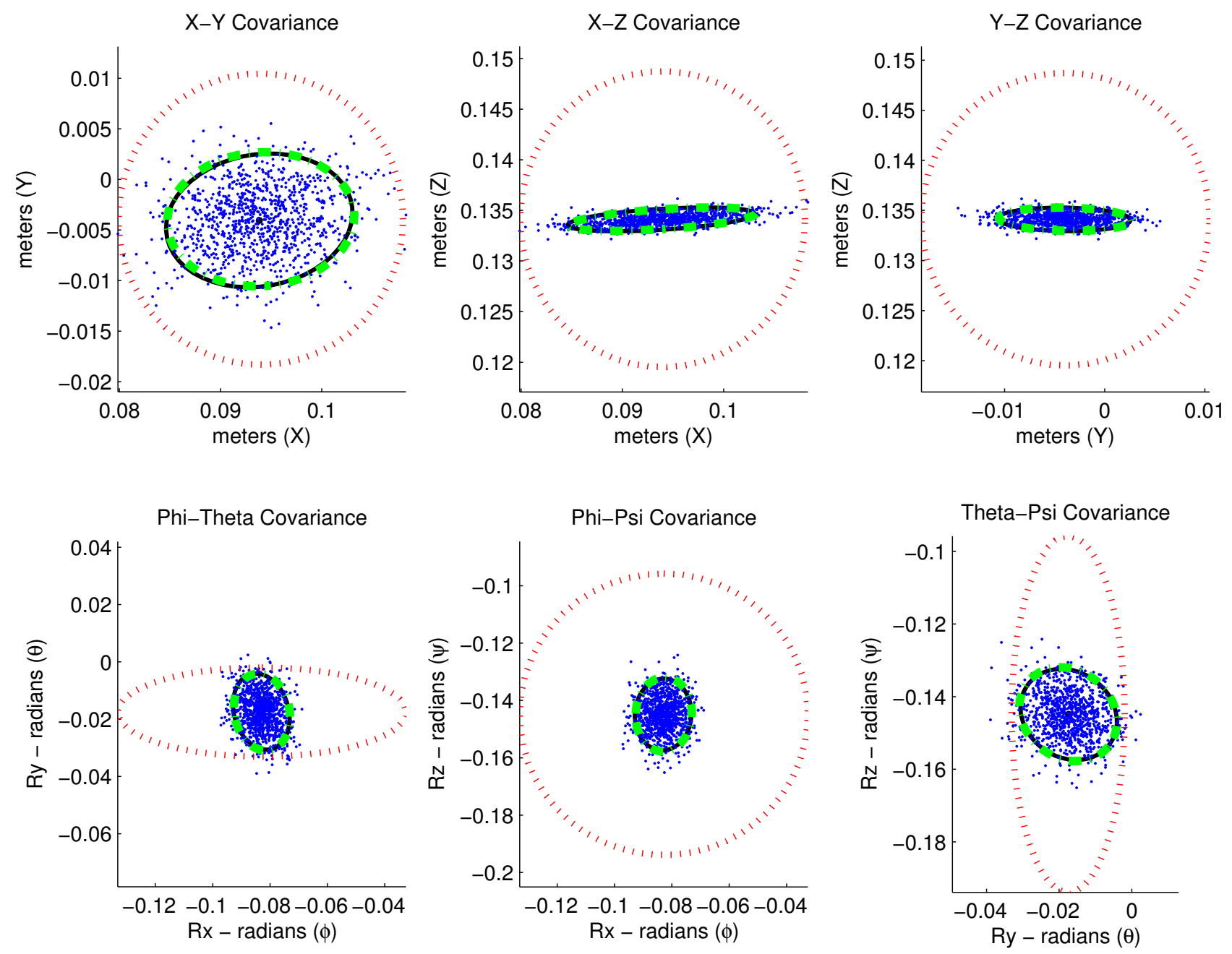

Fig. 3: Covariance projections. The frames show 2D projections of the $6 \mathrm{D}$ covariance hyperellipsoids computed via Monte Carlo simulation (black), via the implicit function (dashed green), and from the accumulation of distance errors (dashed red).

point cloud density. And the second one is an orientation filter, ensuring compatible point correspondence.

After applying ICP to the synthetically generated matching point clouds, each recovered relative pose transformation is plotted as a blue point in Fig. 3. Monte Carlo covariances are plotted in black, while the covariance recovered from the implicit function and the covariance computed by aggregating matching point distances are plotted as a green dashed line and as a red dotted line, respectively. All iso-uncertainty hyperellipsoids have been plotted at a scale of $2 \sigma$.

Building the covariance matrix by aggregating 6 DOF pose distance errors from the matching points, after applying the ICP, and without taking into account the ICP cost function, clearly underestimates the real pose covariance since it does not take into account cross correlations between the various pose variables.

\section{B. Real data object modeling}

A second experiment was performed using a 7 degreesof-freedom WAM robotic arm and a Swissranger ToF camera attached to its gripper. A predefined circular trajectory maintaining the object inside the camera field of view was carried out in order to be able to close the loop needed by the SLAM algorithm. A watering can was approximately placed one meter above the robotic arm reference center in such a way that all viewpoints fall within the arm workspace without passing through ill-posed configurations. One clear advantage of our uncertainty-driven approach is that no precise hand-eye calibration was needed. A set of 20 point clouds were taken during the 360 degrees rotation trajectory at roughly uniformly distributed positions. At each trajectory position, a point cloud was captured and the WAM pose was stored. Note that since no precise hand-eye calibration is 


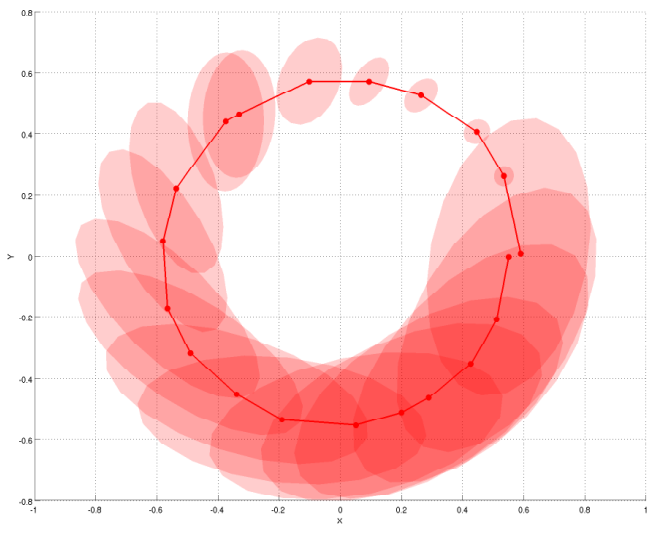

(a) Robot trajectory after all ICP results are aggregated

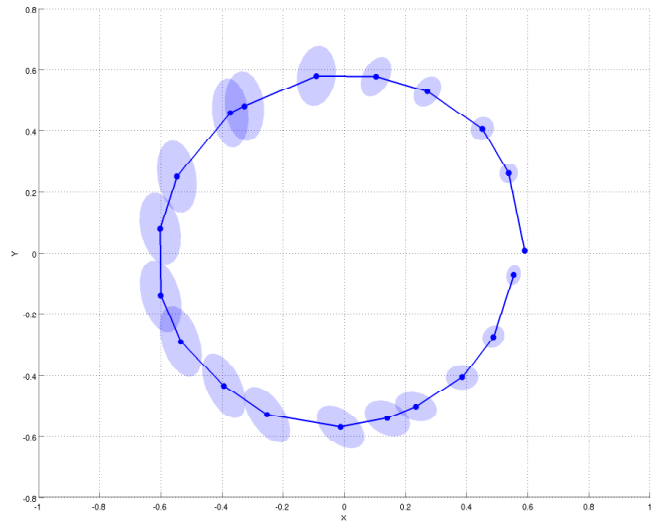

(b) Revised robot trajectory after the loop is closed with the view-based SLAM method

Fig. 4: Robot pose trajectory. Frame a) shows the calculated trajectory and uncertainty estimates after all ICP results are aggregated, but before the loop is closed. Each pose accumulates the estimated error from the previous pose. Frame b) once the loop is closed, uncertainty is reduced and the complete trajectory is corrected.
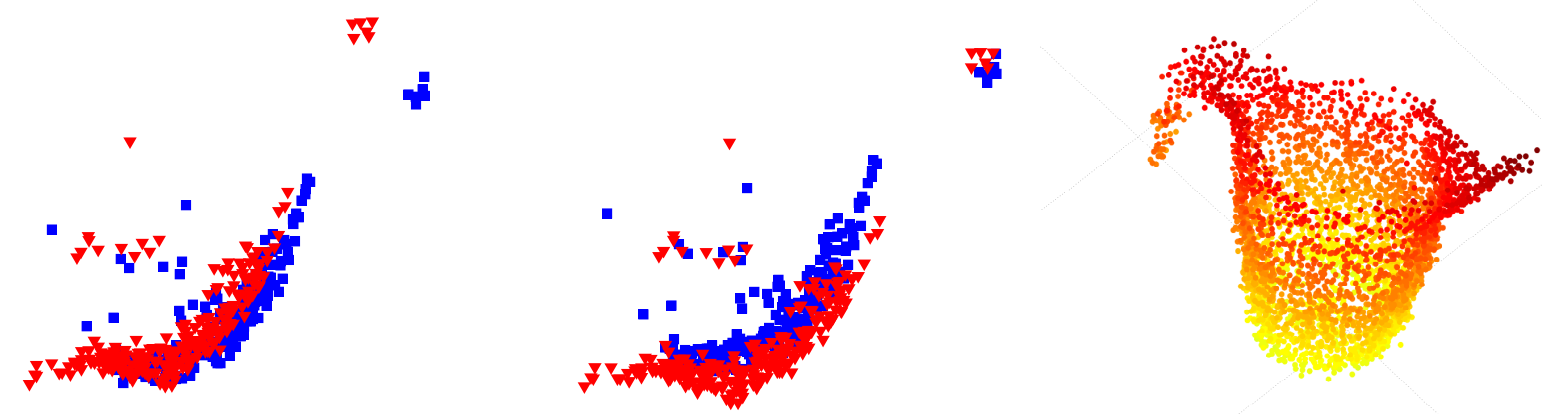

(a) matching of first and last views using aggre-(b) matching of first and last views using gated ICP information-based SLAM

(c) complete 3D model

Fig. 5: The figure shows the advantage of fusing data globally using the proposed filtering scheme. Frames a) and b) contain the sequence's first 3D ToF image (in red) and last (in blue). Data fusion using aggregated ICP accumulates registration error, which is corrected using a globally consistent loop closure provided by the information-based SLAM. Frame c) shows the final complete 3D model.

provided, and since the WAM arm has its own kinematic errors, these poses served only as initialization points for the ICP. In pre-treating raw data, a segmentation algorithm was applied to the incoming point clouds in order to discard outlier object data. The segmentation algorithm consisted of a jump-edge filter and a depth-threshold background filter.

As an example of the proposed method, Fig. 5 shows the final 3D point cloud made for the watering can shown in Fig. 1 . The figure shows the advantage of fusing data globally using the proposed filtering scheme. Frames a) and b) contain the sequence's first 3D ToF image (in red) and last (in blue). Data fusion using aggregated ICP accumulates registration error, which is corrected using a globally consistent loop closure provided by the information-based SLAM. Frame c) shows the final complete 3D model.
Fig. 4 shows the estimated robot trajectories for the cases of ICP relative pose aggregation, and SLAM-based loop closure. After closing the loop, the final trajectory (blue) is closer to a circular shape than not the one achieved purely from accumulating ICP motion estimates (red). The red trajectory tends to describe the typical spiral shape characteristic from error accumulation. In order to get a more precise evaluation of the obtained models, real measures were compared with each model. The real watering can upper edge width is 11.5 centimeters, whereas the aggregated ICP model is 9 centimeters width, and the one obtained after loop closure is enforced is 11.2 centimeters wide. 


\section{COnClusions And Future Research}

This paper presents a method to consistently fuse range images acquired with a ToF camera mounted on a not necessarily calibrated robotic arm to autonomously build a $3 \mathrm{~d}$ object model. Accurate hand-eye calibration is not needed since the method uses globally consistent probabilistic data fusion by means of a view-based information-form SLAM algorithm.

Furthermore, we present a method to linearly propagate noise covariances from the range camera through an error minimization algorithm such as the point to point ICP. The proposed approach, using the implicit theorem, had previously been used in this context only for 2D ICP, and we have derived and extended the method for the $3 \mathrm{D}$ case.

The proposed approach to data fusion for object modeling can be executed in real time, in contrast to iterative methods such as bundle adjustment, provided an efficient approximate nearest neighbor method is in place for the ICP computations. Fortunately, very efficient nearest neighbor search tools exist that take time logarithmic with the number of points in the data set. Efficient globally consistent fusion of pose estimates at loop closure is only possible thanks to the sparsity of the information filtering scheme used.

Foreseen enhancements to the presented method are implicit function propagation of more efficient ICP variants such as the point to plane, plane to plane, and generalized ICP; automatic computation of next viewpoints based on information or entropy minimization metrics; more exhaustive empiric evaluation of the modeling results with a larger set of objects with different shapes and texture; and the application of the built models for the computation of grasping and manipulation directives.

\section{ACKNOWLEDGMENTS}

The authors wish to thank E. Teniente for early implementations of the ICP algorithm and insightful discussions on ICP covariance propagation and R. Valencia for providing us with his view-based SLAM code.

\section{REFERENCES}

[1] S. E. Ghobadi, K. Hartmann, W. Weihs, C. Netramai, O. Loffeld, and H. Roth, "Detection and classification of moving objects-stereo or time-of-flight images," in Proc. Int. Conf. Comput. Intell. Security, vol. 1, Guangzhou, Nov. 2006, pp. 11-16.

[2] S. Hussmann and T. Liepert, "Three-dimensional ToF robot vision system," IEEE Trans. Instrum. Meas., vol. 58, no. 1, pp. 141-146, Jan. 2009.

[3] C. Beder, B. Bartczak, and R. Koch, "A comparison of PMDcameras and stereo-vision for the task of surface reconstruction using patchlets," in Proc. 21st IEEE Conf. Comput. Vision Pattern Recog., vol. 1-8, Minneapolis, June 2007, pp. 2692-2699.

[4] J. Zhu, L. Wang, R. Yang, and J. Davis, "Fusion of time-of-flight depth and stereo for high accuracy depth maps," in Proc. 22nd IEEE Conf. Comput. Vision Pattern Recog., vol. 1-12, Anchorage, June 2008, pp. 3262-3269.
[5] M. Lindner, M. Lambers, and A. Kolb, "Sub-pixel data fusion and edge-enhanced distance refinement for 2D/3D," Int. J. Int. Syst. Tech. App., vol. 5, no. 3-4, pp. 344-354, 2008.

[6] T. Grundmann, Z. Xue, J. Kuehnle, R. Eidenberger, S. Ruehl, A. Verl, R. D. Zoellner, J. M. Zoellner, and R. Dillmann, "Integration of 6D object localization and obstacle detection for collision free robotic manipulation," in Proc. IEEE/SICE Int. Sym. System Integration, Nagoya, Dec. 2008, pp. 66-71.

[7] B. Dellen, G. Alenyà, S. Foix, and C. Torras, "3D object reconstruction from Swissranger sensors data using a spring-mass model," in Proc. 4th Int. Conf. Comput. Vision Theory and Applications, vol. 2, Lisbon, Feb. 2009, pp. 368-372.

[8] J. U. Kuehnle, Z. Xue, M. Stotz, J. M. Zoellner, A. Verl, and R. Dillmann, "Grasping in depth maps of time-of-flight cameras," in Proc. Int. Workshop Robotic Sensors Environments, Otawa, Oct. 2008, pp. $132-137$

[9] A. Saxena, L. Wong, and A. Y. Ng., "Learning grasp strategies with partial shape information," in Proc. 23th AAAI Conf. on Artificial Intelligence, Chicago, Jul. 2008, pp. 1491-1494.

[10] B. Triggs, P. McLauchlan, R. Hartley, and A.W.Fitzgibbon, "Bundle adjustment - a modern synthesis," in Proc. Int. Workshop on Vision Algorithms, ser. Lect. Notes Comput. Sci., vol. 1883, Corfu, Sep. 1999, pp. 153-177.

[11] F. Dellaert, S. Seitz, C. Thorpe, and S. Thrun, "Structure from motion without correspondence," in Proc. 14th IEEE Conf. Comput. Vision Pattern Recog., vol. 2, Head Island, Jun. 2000, pp. 557-564.

[12] M. W. M. G. Dissanayake, P. Newman, S. Clark, H. F. Durrant-Whyte, and M. Csorba, "A solution to the simultaneous localization and map building (SLAM) problem," IEEE Trans. Robot. Automat., vol. 17, no. 3, pp. 229-241, Jun. 2001.

[13] V. Ila, J. M. Porta, and J. Andrade-Cetto, "Information-based compact Pose SLAM," IEEE Trans. Robot., vol. 26, no. 1, pp. 78-93, Feb. 2010.

[14] A. Kolb, E. Barth, and R. Koch, "ToF-sensors: New dimensions for realism and interactivity," in Proc. IEEE CVPR Workshops, vol. 1-3, Anchorage, June 2008, pp. 1518-1523.

[15] P. Besl and N. McKay, "A method for registration of 3D shapes," IEEE Trans. Pattern Anal. Machine Intell., vol. 14, no. 2, pp. 239-256, Feb. 1992.

[16] Y. Chen and G. Medioni, "Object modeling by registration os multiples ranges images," in Proc. IEEE Int. Conf. Robot. Automat., vol. 3, Sacramento, Apr. 1991, pp. 2724-2729.

[17] Z. Zhang, "Iterative point matching for registration of free-form curves and surfaces," Int. J. Comput. Vision, vol. 13, pp. 119-152, 1994.

[18] S. Rusinkiewicz and M. Levoy, "Efficient variants of the ICP algorithm," in Proc. 3rd Int. Conf. 3D Digital Imaging Modeling, Quebec, May 2001, pp. 145-152.

[19] J. Minguez, L. Montesano, and F. Lamiraux, "Metric-based iterative closest point scan matching for sensor displacement estimation," IEEE Trans. Robot., vol. 22, no. 5, pp. 1047-1054, Oct. 2006.

[20] J. Clarke, "Modelling uncertainty: A primer," University of Oxford. Dept. Engineering science, Tech. Rep. 2161/98, 1998.

[21] R. Valencia, E. Teniente, E. Trulls, and J. Andrade-Cetto, "3D mapping for urban serviece robots," in Proc. IEEE/RSJ Int. Conf. Intell. Robots Syst., Saint Louis, Oct. 2009, pp. 3076-3081.

[22] R. Smith, M. Self, and P. Cheeseman, "Estimating uncertain spatial relationships in robotics," in Autonomous Robot Vehicles, 1990, pp. $167-193$

[23] M. Kaess, A. Ranganathan, and F. Dellaert, "iSAM: Incremental smoothing and mapping," IEEE Trans. Robot., vol. 24, no. 6, pp. 1365-1378, 2008.

[24] T. Davis, J. Gilbert, S. Larimore, and E. Ng, "A column approximate minimum degree ordering algorithm," ACM T. Math. Software, vol. 30, no. 3, pp. 353-376, 2004.

[25] F. Dellaert and M. Kaess, "Square root SAM: Simultaneous localization and mapping via square root information smoothing," Int J. Robot. Res., vol. 25, no. 12, pp. 1181-1204, 2006. 